activation of canonical and noncanonical NF- $\kappa \mathrm{B}$ signalling, and increased the expression of inflammatory cytokines and adhesion molecules, which could be blocked by targeting NIK. Conclusions These findings suggest that in addition to regulating noncanonical signalling, NIK can serve as an amplifier of canonical NF- $\kappa \mathrm{B}$ signalling and associated inflammatory responses in EC, which may play a role in development and maintenance of chronic inflammation. Consequently, NIK may be an attractive therapeutic target.

Disclosure of interest P. Kucharzewska Employee of: Employed by AstraZeneca, K. Jeucken: None declared, C. Maracle: None declared, J. P. van Hamburg: None declared, H. Olsson Employee of: Employed by AstraZeneca, S. Tas: None declared

\section{SMOC2 HAS DIFFERENTIAL EFFECTS ON CARTILAGE AND BONE FORMATION}

${ }^{1} \mathrm{~T}$ Peeters*, ${ }^{1} \mathrm{R}$ Lories, ${ }^{2} \mathrm{~F}$ Cailotto. ' $S$ keletal Biology and Engineering Research Centre Dept. Development and Regeneration, KU LEUVEN, Leuven, Belgium; ${ }^{2}$ MOPA, Université de Lorraine, Nancy, France

\subsection{6/annrheumdis-2018-EWRR2018.5}

Introduction SMOC2, a secreted calcium-binding protein of the BM-40/SPARC family was identified from a chondrogenic extract of articular cartilage and is increased in osteoarthritic cartilage. Alterations in bone and cartilage resulting from dysregulated signalling pathways are a hallmark of osteoarthritis.

Objectives To evaluate the role of SMOC2 in in vitro osteogenesis and chondrogenesis and its interaction with Wnt signalling.

Methods Wild-type Smoc2 (Smoc2+) or Smoc2 lacking the calcium binding domain (?CaBD) were stably overexpressed and wild-type Smoc2 was silenced (Smoc2-). Alkaline phosphatase (ALP) activity assay and Alizarin Red staining were performed in MC3T3 cells and human periosteal derived cells (hPDCs). Proteoglycan content and mineralization were assessed in ATDC5 micromasses by Alcian blue and Alizarin Red staining. Mice femoral head caps (FHC) were stimulated with recombinant IL-1b and SMOC2. Cartilage loss was evaluated by DMMB assay and Safranin O staining. TCF1 levels were analysed by immunohistochemistry. Human articular chondrocytes (hACs) were stimulated with recombinant SMOC2. Gene expression of osteoblast markers (Opn, Osx, Col1a2), chondrogenic markers (Acan, Col2a1, Col10a1, Mmp13) and canonical Wnt target genes (Axin2, Lef1) were analysed by qPCR and Wnt signalling by Western blot.

Results Alizarin red staining and ALP activity were reduced in Smoc2 + MC3 T3 cells. Osteoblast gene expression levels were altered in Smoc2 + cells. ?CaBD cells and calcium supplementation to Smoc2 +showed a partial rescue of the effects of Smoc $2+$ cells. We validated these results in hPDCs. We could not observe an effect on osteogenesis when silencing Smoc2. Smoc2 +ATDC5 micromasses showed reduced chondrogenic gene expression and Alcian blue and Alizarin Red staining intensity. We observed a reverse pattern with an increased chondrogenic gene expression in Smoc2- ATDC5 micromasses. Smoc2 + and Smoc2- ATDC5 cells altered canonical Wnt signalling in opposite directions. SMOC2 also alters protein levels of CamKII. IL-1b triggered cartilage loss was increased and TCF1 levels were reduced in mice FHC stimulated with recombinant SMOC2. In hACs, we observed a negative effect of SMOC2 on chondrocyte markers and canonical Wnt target genes.
Conclusions Our results suggest an inhibitory role of Smoc2 on mineralization. The inhibitory effects on mineralization are at least partially regulated through interaction with calcium. Moreover, Smoc2 is a regulator of chondrogenic differentiation and cartilage homeostasis by regulating canonical and non-canonical Wnt signalling.

Disclosure of interest None declared

\section{LUMICAN: A NOVEL GYCOPROTEIN MEDIATING INFLAMMATION IN OSTEOARTHRITIS}

${ }^{1} \mathrm{G}$ Barreto*${ }^{*}{ }^{2} \mathrm{~B}$ Senturk, ${ }^{1} \mathrm{~L}$ Colombo, ${ }^{3} \mathrm{P}$ Neidenbach, ${ }^{3} \mathrm{G}$ Salzmann, ${ }^{2} \mathrm{M}$ Rottmar, ${ }^{1} \mathrm{M}$ Zenobi-Wong. ${ }^{1}$ ETH Zurich, Zurich; ${ }^{2}$ Swiss Federal Laboratories for Materials Science and Technology, Dübendorf; ${ }^{3}$ Schulthess Klinik, Zurich, Switzerland

10.1136/annrheumdis-2018-EWRR2018.6

Introduction Lumican (LUM) is major extracellular matrix protein which is present in glycoprotein format in adult articular cartilage. Recently, LUM was show to augment pathogen-associated molecular patterns (PAMPs) activation of the TLR4 signalling cascade. Given that TLR4 is highly associated with inflammation in rheumatic disease, we aim to decipher the LUM role in Osteoarthritis and TLR4 associated inflammation. Objectives To measure LUM in synovial fluid from patients with arthritic conditions and to study the role of LUM on TLR4 activation in osteoarthritis.

Methods Synovial fluid (SF) was obtained from knee meniscus tear $(n=11)$, first carpometacarpal (CMC-I) OA $(n=11)$ and knee OA $(n=40)$ patients. LUM glycoprotein levels were analysed by enzyme-linked immunosorbent assay (ELISA). Human monocytes were isolated from healthy individuals and differentiated into M1-like and M2-like macrophages by lipopolysaccharide (LPS) and interleukin-4, respectively. LUM (1 ug/ml) was added either together with macrophage polarisation inducers or alone. Primary chondrocytes and OA cartilage explants were stimulated for 24 hour with LUM $(1 \mu \mathrm{g} / \mathrm{ml})$, LPS $(10 \mathrm{ng} / \mathrm{ml})$, or a combination of both. Conditioned media was analysed for selected secreted molecules by xMAP technology. Macrophages surface expression markers CD197 and CD206 were analysed by Fluorescence-Activated Cell Sorting (FACS). Cartilage explants were immunofluorescently double stained for collagen type II and X.

Results LUM glycoprotein levels were significantly upregulated in knee OA SF vs. controls. In normal articular chondrocytes, LUM combined with LPS caused an upregulated secretion of catabolic markers characteristic of OA such as IL-6, MMP-1, and MMP-13 in comparison to LPS stimulation. LUM alone had no observable effects. A similar response occurred in cartilage explant cultures, with increased MMP-1 secretion levels and marked cartilage degradation with the LPS/LUM combination. Interestingly, LUM stimulation with the polarisation inducer, LPS for M1, and IL-4 for M2 macrophages, upregulated M1 macrophage secretion of TNF-alpha and downregulated IL-10 secretion in M2 macrophages relative to control and LUM alone. FACS results followed the same trend, with increased CD197 expression in LUM/LPS combination vs. LUM alone or control, and downregulation of CD206 expression when LUM/IL-4 combination vs. LUM alone or control. Conclusions The results presented here show that LUM is highly upregulated in SF of OA and RA patients. In addition, we observed that TLR-expressing chondrocytes do not respond to LUM, however LUM augmented the LPS-induced TLR4 signalling cascade and consequently the catabolic response. 
Moreover, we also demonstrate the ability of LUM to upregulate M1 macrophage and downregulate M2 macrophage polarisation. Our findings strongly support a pathogenic role of LUM, as mediator of PAMP-induced TLR-4 activation of inflammation, cartilage degradation and macrophage polarisation in OA and other rheumatic diseases.

Disclosure of interest None declared

\section{ACPA-INDUCED MOBILITY OF PRIMED SYNOVIAL FIBROBLASTS: THE MISSING LINK BETWEEN ACPA- INDUCED BONE LOSS AND SYNOVIAL CHANGES}

${ }^{1} \mathrm{M}$ Sun*, 'B Rethi, ${ }^{1} \mathrm{~V}$ Joshua, ${ }^{1} \mathrm{~A}$ Krishnamurthy, ${ }^{1} \mathrm{~A}$ Hensvold, ${ }^{2} \mathrm{SB}$ Catrina, ${ }^{3} \mathrm{C}$ Ospelt, ${ }^{1} \mathrm{~V}$ Malmström, ${ }^{1} \mathrm{~J}$ Steen, ' $\mathrm{M}$ Engström, ${ }^{1} \mathrm{H}$ Wähämaa, ${ }^{1} \mathrm{Al}$ Catrina. ${ }^{1}$ Rheumatology unit, Department of Medicine; ${ }^{2}$ Department of Molecular Medicine and Surgery, Karolinska Institute, Stockholm, Sweden; ${ }^{3}$ Centre of Experimental Rheumatology, Department of Rheumatology, University Hospital Zürich, Zurich, Swaziland

\subsection{6/annrheumdis-2018-EWRR2018.7}

Introduction Anti-citrullinated proteins antibodies (ACPAs) injected in mice induce IL-8 dependent bone loss and arthralgia, but no synovial changes. We hypothesised that additional stimuli, sensitising the synovial compartment to ACPA effects, might be needed for the transition from bone to synovial pathology.

Methods Fibroblast like synoviocytes (FLSs) were isolated from synovial tissue biopsies obtained from RA patients. Polyclonal ACPA and other non-ACPA IgGs were separated from peripheral blood of RA patients by affinity purification on protein $G$ and cyclic citrullinated peptide (CCP) -2 columns. Monoclonal ACPAs were cloned from synovial fluid B-cells. FLS migration was tested by scratch-assays using IncuCyte (Essen bioscience). SF adhesion was analysed by xCELLigence System Real-Time Cell Analyzer (ACEA bioscience). Peptidylarginine deiminases (PAD) expression and protein citrullination were evaluated by immunohistochemistry and immunofluorescent stainings.

Results FLS mobility was not affected by polyclonal ACPA stimulation. However, exposing the cells to IL-8 or to a transient serum starvation increased PAD expression and the amount of citrullinated proteins in the cells. In line with these findings, starved FLSs obtained sensitivity to ACPAs and responded with an increased mobility to antibody stimulation. Similar effects were observed in the presence of three out of ten monoclonal ACPAs, suggesting that only APCAs with certain fine specificity could target FLSs. The ACPA-induced migration was abolished by pre-incubating the cells with PAD inhibitor or by combining the ACPAs with citrullinated but not native fibrinogen. IL-8 alone could not influence fibroblast migration but it synergistically increased the response to ACPAs. The role for inflammatory stimuli in sensitising FLSs to ACPA binding was further supported by the labelling of synovial tissues of RA patient, but not of healthy controls, using monoclonal ACPAs.

Conclusions We demonstrated that FLS response to ACPA stimulation was enabled by stress- or cytokine-induced citrullination in the cells. These results suggest an important role for transient synovial insults in setting the stage for the ACPAmediated transition towards chronic synovitis.

Disclosure of interest None declared

\section{ALTERED LYMPH NODE STROMAL CELLS DURING THE EARLIEST PHASES OF RHEUMATOID ARTHRITIS}

${ }^{1} \mathrm{C}$ Ospelt, ${ }^{1} \mathrm{E}$ Karouzakis, ${ }^{2} \mathrm{~J}$ Hähnlein, ${ }^{2} \mathrm{H}$ Semmelink, ${ }^{1} \mathrm{R}$ Gay, ${ }^{2,3} \mathrm{P}-\mathrm{P}$ Tak, ${ }^{2,4} \mathrm{D}$ Gerlag, ${ }^{1} S$ Gay, ${ }^{2} \mathrm{~L}$ Van Baarsen*. 'Center of Experimental Rheumatology, University Hospital of Zurich, Zurich, Switzerland; ${ }^{2}$ Clinical Immunology and Rheumatology, Academic Medical Centre/University of Amsterdam, Amsterdam, Netherlands; ${ }^{3}$ Current: GlaxoSmithKline, Stevenage; ${ }^{4}$ Current: Clinical Unit Cambridge, GlaxoSmithKline, Cambridge, UK

\subsection{6/annrheumdis-2018-EWRR2018.8}

Introduction Rheumatoid arthritis (RA) is an autoimmune disease with unknown etiopathogenesis where systemic autoimmunity precedes clinical disease onset. Adaptive immunity is initiated in lymphoid tissue where lymph node stromal cells (LNSC) play a crucial role in shaping the immune response and maintaining peripheral tolerance.

Objectives We developed an experimental model for studying the functional capacities of human LNSC during the earliest phases of RA and compared their cellular and molecular characteristics to LNSC from healthy volunteers.

Methods ACPA + RA patients $(\mathrm{n}=24)$, ACPA + RA risk individuals $(n=23)$ and seronegative healthy controls $(n=14 ; H C)$ underwent ultrasound-guided inguinal lymph node biopsy. Human LNSCs were isolated and expanded in vitro for cellular (flow cytometry), molecular (methylome, transcriptome and microRNA) and functional analyses.

Results Key LN chemokines CCL19, CCL21 and CXCL13 were induced in LNSCs upon stimulation with TNF $\alpha$ and lymphotoxin $\alpha 1 \beta 2$, but to a lesser extent in LNSCs from RA patients. RNA sequencing was performed on LNSC of HC $(\mathrm{n}=5)$, ACPA +RA risk individuals $(\mathrm{n}=6)$ and ACPA +RA patients $(n=4)$. Of interest, LNSC from ACPA +RA risk individuals and ACPA + RA patients revealed a common significantly differential expressed gene signature compared with $\mathrm{HC}$ LNSC. Pathway analysis of this common signature showed, among others, significant enrichment of pathways affecting actin cytoskeleton, focal adhesion and cell junction. Accordingly, in a gel contraction assay LNSC from ACPA + RA risk individuals and RA patients showed impaired collagen contraction compared to healthy LNSC. In RA LNSC a significant enrichment was observed for genes involved in TGFb signalling while in RA-risk LNSC cell cycle genes were differentially expressed compared with HC. DNA methylation analyses revealed common differentially methylated $\mathrm{CpG}$ sites (DMS) in LNSC from ACPA + RA patients $(n=5)$ and ACPA + RA risk individuals $(n=3)$ compared with HC $(n=4)$. These DMS were significantly hypomethylated and associated with antigen processing and presentation (HLA-DRB1).

Conclusions This data point towards alterations in the cytoskeleton and antigen-processing and presentation in LNSC from ACPA+RA risk individuals and RA patients. Further studies are required to investigate the influence of this LNSC abnormality on immune responses.

Disclosure of interest C. Ospelt: None declared, E. Karouzakis: None declared, J. Hähnlein: None declared, H. Semmelink: None declared, R. Gay: None declared, P.-P. Tak Employee of: Currently: Senior Vice President $\mathrm{R}$ and D Pipeline, Global Development Leader and Chief Immunology Officer, GSK, D. Gerlag Employee of: Currently: Head Clinical Unit Cambridge at GSK, S. Gay: None declared, L. Van Baarsen: None declared 